\title{
ESCALA DE TOMA DE DECISIONES PARA DIRECTIVOS
}

\author{
Cynthia Zaira Vega Valero, Diana Elisabeth Reza Morales, Oscar García Arreola, Rosa Alejandra \\ Hernández Toledano y David Ruiz Méndez \\ Facultad de Estudios Superiores Iztacala, UNAM \\ México
}

\begin{abstract}
RESUMEN
En la investigación organizacional se ha mostrado que la efectividad de un directivo y el éxito organizacional se relaciona con la toma de decisiones. Pese a ello, aún no se cuenta con un instrumento que evalúe la toma de decisiones en las organizaciones y que se enfoque en habilidades directivas. El objetivo de esta investigación es presentar el desarrollo de la escala de toma de decisiones enfocada en el ámbito laboral y en habilidades directivas, así como presentar evidencia de la confiabilidad y validez de la medición. Se hizo un estudio transversal con la participación de 235 directivos mexicanos. Se efectuaron un análisis de consistencia interna y un análisis factorial exploratorio. Los resultados mostraron un valor de confiabilidad e índices de ajuste aceptables. A partir del análisis factorial se obtuvo una estructura con tres factores: 1 ) identificación y análisis de información y alternativas; 2 ) identificación de objetivos organizacionales, y 3 ) decisiones precipitadas. Se concluye que la escala demuestra propiedades psicométricas adecuadas y que se puede considerar como un medio para proporcionar mediciones de toma de decisiones en las organizaciones en directivos.
\end{abstract}

Palabras Clave:

toma de decisiones, directivos, organizaciones, análisis factorial exploratorio

\section{DECISION-MAKING SCALE FOR MANAGERS}

\begin{abstract}
Organizational research has shown that an effective managerial performance and organizational success is related to decision making. However, a measurement instrument to assess organizational decision-making focused on managerial abilities is not yet available. The aim of this study is to present a decision-making scale designed for work settings and focused on managerial skills, as well as to show evidence of its reliability and statistical validity. A cross-sectional study was carried out with 235 Mexican managers. Internal consistency analysis, and an exploratory factor analysis were performed. Results showed acceptable reliability values, and adequate factor exploration conditions. Factor analysis revealed a three-factor structure: Identification and analysis of the alternatives, identification of organizational objectives and hasty decisions. It is concluded that the scale shows adequate psychometric properties and can be considered as means of measuring organizational decision-making in managers.
\end{abstract}

Keywords:

decision making; managers, organizations, exploratory factor analysis

Bitácora del ARtículo:

| Recibido: 1 de Septiembre de 2020 | Aceptado: 07 enero de 2021 | Publicado en línea: Enero - Junio de 2021 | 


\title{
Autoría y Derechos de Propiedad Intelectual
}

\section{ESCALA DE TOMA DE DECISIONES \\ PARA DIRECTIVOS}

\author{
Cynthia Zaira Vega Valero, Diana Elisabeth Reza Morales, Oscar García Arreola, Rosa Alejandra \\ Hernández Toledano y David Ruiz Méndez \\ Facultad de Estudios Superiores Iztacala, UNAM \\ México
}

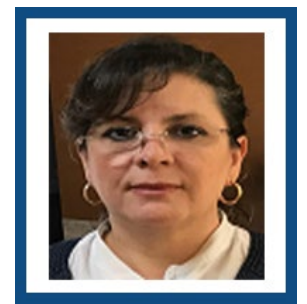

Cynthia Zaira Vega Valero

FES- Iztacala, UNAM

Correo: vegavalero@hotmail.com

Imparte clases en licenciatura y posgrado en la FES Iztacala de la UNAM; es responsable de la maestría en Psicología: Residencia en Gestión Organizacional. Es tutora de maestría y doctorado; es colaboradora de comités de admisión del posgrado de Psicología de aspirantes para ingreso a maestría y doctorado.

\section{CONTRIBUCIÓN DE LOS AUTORES}

Diana Elisabeth Reza Morales FES- Iztacala, UNAM

Correo:psic.diana.reza.morales@gmail. com

Licenciada en Psicología y maestra en Psicología con residencia en Gestión Organizacional por parte de la UNAM. Tiene experiencia laboral en el área de recursos humanos, en particular en reclutamiento y selección de personal de puestos operativos y administrativos, capacitación, evaluación de desempeño y desarrollo organizacional. Ha impartido clases a nivel licenciatura con materias relacionadas con el ámbito organizacional, como Psicología empresarial, Administración de pruebas psicométricas y Procesos de reclutamiento y Selección de personal. Imparte clases a nivel posgrado en la FES Iztacala.

\begin{abstract}
Cynthia Zaira Vega Valero se encargo de la dirección para la constitución del artículo. Recopilación y análisis de artículos empíricos y documentos teóricos para la construcción del marco teórico, redacción del artículo, análisis exhaustivo de datos y la discusión. | Diana Elisabeth Reza Morales realizó la búsqueda, recopilación y análisis de artículos empíricos y documentos teóricos en bases de datos para el apoyo en la construcción del marco teórico, redacción del artículo, recolección y apoyo en el análisis de datos. I Oscar García Arreola apoyó para la construcción y delimitación del marco teórico, redacción del artículo, recolección y el exhaustivo análisis de datos. | Rosa Alejandra Hernández Toledano apoyó para la construcción del marco teórico, redacción del artículo involucrando precisiones en el formato APA, recolección y apoyo en el análisis de datos. - I David Ruiz Méndez participó en la elaboración del abstract, construcción del marco teórico, redacción del artículo y el análisis exhaustivo de datos.
\end{abstract}

\section{AGRADECIMIENTOS}

La elaboración de este artículo fue posible gracias al financiamiento correspondiente al proyecto PAPIIT-UNAM IT300218.

\section{Datos de Filiación de los Autores}

Facultad de Estudios Superiores Iztacala, UNAM

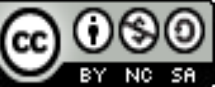

Copyright: @ 2020 Vega-Valero, C.Z.; Reza-Morales, D.E.; García-Arreola, O.; Hernández-Toledano, R.A.; \& Ruiz-Méndez, D.

Este es un artículo de acceso abierto distribuido bajo los términos de la licencia Creative Commons Reconocimiento-NoComercial 4.0 Internacional, por lo que su contenido gráfico y escrito se puede compartir, copiar y redistribuir total o parcialmente sin necesidad de permiso expreso de sus autoras con la única condición de que no se puede usar con fines directamente comerciales y los términos legales de cualquier trabajo derivado deben ser los mismos que se expresan en la presente declaración. La única condición es que se cite la fuente con referencia a la Revista Digital Internacional de Psicología y Ciencia Social y a sus autoras. 


\section{TABLA DE CONTENIDO}

MÉTODO

Participantes, 26

Tipo de investigación, 26

PROCEDIMIENTO

Mediciones, 27

ANÁLISIS ESTADÍSTICOS

RESULTADOS

Discusıón

CONCLUSIONES

SUGERENCIAS

REFERENCIAS 
$\mathbf{L}$ as habilidades directivas no son atributos de personalidad o tendencias estilísticas, sino que consisten en conjuntos identificables y observables de acciones que los individuos efectúan en las organizaciones (Analoui, Labbaf y Noorbaksh, 2000; Sánchez, Aguirre, Barrales, Vergara y Mota, 2015). Son conductas que pueden ser entrenadas y practicadas, a tal grado que los propios individuos pueden demostrar, mejorar o perfeccionarlas con el objetivo de tener un efecto significativo en su desempeño dentro de una organización (VandenBos, 2007; Whetten y Cameron, 2011).

En la literatura se destaca un conjunto de habilidades centrales que debe tener un directivo para ser efectivo en su puesto, tales como solución de problemas (Pereda, López y González, 2014; Ramón, Ollage, Granda y Naranjo, 2017; Rodríguez y Posadas, 2005; Vega-Valero, Hernández-Toledano, García-Arreola, Nava-Quiroz y Ruiz-Méndez, 2019), habilidades sociales (Moreno-Jiménez, Blanco-Donoso, Aguirre-Camacho, De Riva y Herrero, 2014; Salvador, Fuente y Álvarez, 2009; Veloso-Besio, Cuadra-Peralta, Gil-Rodríguez, Quiroz-Cornejo y Meza-Castro, 2015), gestión del tiempo (Salazar, 2017; Vickers, Dowell y House, 2019), gestión de trabajo (Pérez, Barroso, Escalante y Eliseo, 2008; Sánchez et al., 2015; Whetten y Cameron, 2011) e involucramiento en el proceso creativo (Quintero, Granada, Álvarez y Calderón, 2011). De entre estas variables, una habilidad clave es la toma de decisiones (Griffin, y Van Fleet, 2016; Kepner, y Tregoe, 1972; Madrigal, 2009; Vélez, 2006; Whetten, y Cameron, 2011).

En el contexto organizacional se indica que cuando un directivo se involucra en un patrón de toma de decisiones estratégico y sistemático, no sólo produce beneficios para la organización sino que establece una ventaja competitiva en relación con otras organizaciones (Bruine de Bruin, Parker y Fischhoff, 2012). Por otro lado, una toma de decisiones asistemática puede generar situaciones desfavorables en distintos niveles de la organización (Cabeza de Vergara, Muñoz y Vivero, 2004). Tomar una decisión implica elegir el mejor curso de acción o alternativa basándose en la información que se tiene en ese momento (Anderson, et al., 2016; Butler, 1991; Peñaloza, 2010; Winterfeldt, y Edwards, 1986). En la literatura la toma de decisiones se considera como un proceso susceptible a analizarse a partir de los siguientes aspectos: 1) definición del problema; 2) identificación de posibles alternativas; 3 ) identificación de las consecuencias de cada alternativa; 4) evaluación de al- ternativas en relación con un objetivo; 5) evaluación de la probabilidad de que ocurra una u otra consecuencia, y 6) elección de la mejor opción (Anderson et al., 2016; Butler, 1991; Furby y Beyth-Marom, 1992). A pesar de la identificación de los aspectos, la manera en que se ha medido esta variable es con base en los resultados (Freixa, 2003). Esto significa que la decisión se evalúa de manera indirecta y en un solo momento, perdiendo la posibilidad de medir las características del proceso de acuerdo con los pasos planteados y limitando la indagación empírica (Winterfeldt y Edwards, 1986).

Otras posturas que abordan la toma de decisiones han desarrollado una serie de escalas que abordan la toma de decisiones retomando los aspectos anteriores e incluyendo otros elementos, como el estilo decisorio analítico o intuitivo (Azzollini y Depaula, 2013; Sjöberg, 2003), el riesgo de una consecuencia negativa de la decisión que tienen las personas, la posibilidad de una consecuencia positiva, y saber si la decisión se encontraba bajo el control del decisor (Sjöberg, 2003), la valoración positiva o negativa del individuo respecto a las consecuencias futuras a partir de sus rasgos de personalidad (Nenkov, Inman y Hulland, 2008) y el patrón de comportamiento decisorio ante un conflicto (Mann, Burnett, Radford y Ford, 1997; Maciá, et al., 1996). Sin embargo, los instrumentos indicados se enfocan en medir la toma de decisiones en un contexto diferente al organizacional. Además esta habilidad se mide de manera genérica a partir de la apreciación de uno mismo. Esto resulta en limitaciones importantes para la identificación de las conductas específicas que tiene o debe desarrollar un directivo para caracterizar su toma de decisiones.

Uno de los modelos teóricos para explicar la toma de decisiones a partir de habilidades es el de toma de decisiones proactivas (TDP) de Siebert y Kunz (2016). Este modelo explica la toma de decisiones como un concepto multidimensional. Parte de su explicación se relaciona con competencias conductuales como la identificación de objetivos, alternativas, información y anticipación de consecuencias. Adicionalmente, este modelo supone que la personalidad se relaciona con la toma de decisiones, identificando dos estilos: 1) la toma dediciones proactiva, 2) y la toma de decisiones reactiva. Por ejemplo, un individuo con personalidad proactiva establece metas que puede cumplir en cualquier contexto, mientras que los individuos con personalidad reactiva suelen ser pasivos y están limitados por su entorno. A partir de este modelo se propuso un instrumento conformado por 21 ítems con opciones de respuesta que van en un continuo de 1 ("muy en desacuerdo") a 7 ("muy de acuerdo"). Los ítems estaban distribuidos en cuatro dimensiones de acuerdo con una habilidad: 1) 
identificación sistemática de objetivos (tres ítems); 2) identificación sistemática de alternativas (cuatro ítems); 3) búsqueda sistemática de información (tres ítems), y 4) uso del radar decisional (cinco ítems); además de dos dimensiones para medir los rasgos de personalidad (seis ítems). El valor de confiabilidad reportado por los autores del instrumento fue de 0.840. Adicionalmente se reportó evidencia de validez de la medición (Siebert y Kunz, 2016).

A pesar de las ventajas que ofrece el instrumento de Siebert y Kunz (2016) en términos de la medición de habilidades relativas a la toma de decisiones, éste no fue diseñado para un contexto organizacional ni está enfocado a directivos. Lo anterior genera una serie de problemas al usar el instrumento para medir la toma de decisiones en las organizaciones. Por ejemplo, en el contexto organizacional se considera que la personalidad de un directivo no es necesariamente la característica más importante en su desempeño, sino sus habilidades en un contexto complejo y demandante (Judge, Bono, Ilies y Gerhardt, 2002; Rynes, Colbert y Brown, 2002). Por tanto, las dimensiones de personalidad del instrumento original podrían no ofrecer información relevante de la toma de decisiones en directivos. Otro aspecto a considerar se relaciona con las opciones de respuesta que presenta el instrumento. Dichas opciones están redactadas en términos de acuerdo-desacuerdo, por lo que esta manera de medir la toma de decisiones no permite identificar la valoración de un directivo en términos de la frecuencia del ejercicio de sus habilidades de toma de decisiones.

Considerando estas limitaciones, se retomaron sólo las dimensiones del instrumento que aludían estrictamente a las habilidades. Dichas dimensiones fueron la identificación de objetivos y alternativas, la búsqueda de información y la anticipación de consecuencias. Fue a partir del tipo de comportamientos sugeridos por estas dimensiones que se elaboraron los ítems de la escala. Por otro lado, se decidió excluir las dimensiones que evaluaban la personalidad en el proceso de construcción de la escala, ya que el interés se encuentra en la frecuencia del ejercicio de habilidades de toma de decisiones en un contexto organizacional. De acuerdo con lo anterior, se desarrolló una escala que tuviera el propósito de evaluar la frecuencia de las habilidades de toma de decisiones en contexto organizacional y con énfasis en directivos. La escala propuesta se titula "Escala de toma de decisiones en directivos" (ETDD) (anexo 1). El desarrollo de la escala siguió un proceso de validación de contenido (Ruiz y Vega, 2018). Sin embargo, durante el desarrollo de un instrumento de medición fue necesario obtener evidencia de la confiabilidad y validez de las mediciones, por lo que el objetivo de la presente investigación es presentar evidencia de la confiabilidad y validez de la ETDD, además de buscar estimar la confiabilidad a partir de la consistencia interna de la medición y la validez por medio de la exploración factorial (DeVellis, 2017; Kyriazos y Stalikas, 2018).

\section{Método}

\section{Participantes}

En el estudio participaron 235 directivos mexicanos de manera voluntaria. El muestreo utilizado fue de tipo no probabilístico por conveniencia (Hernández, Fernández-Collado y Baptista, 2014).

De la muestra, 60\% (141) eran hombres y 40\% (94) mujeres con un nivel de edad de 21 a 69 años. La mayoría tenía un nivel escolar de licenciatura o ingeniería $(46 \% ; 108)$, seguido por un nivel de posgrado $(24.7 \%$; $58)$, bachillerato $(15.7 \% ; 37)$, carrera técnica $(8.5 \% ; 20)$ y secundaria $(4.7 \% ; 12)$. Del total, $27.2 \%$ (64) correspondía a mandos altos, $62.1 \%$ (146) a mandos medios y $10.6 \%$ (25) a mandos bajos. El 53.2\% (125) se desempeñaba en organizaciones públicas y $46.8 \%$ (110) en privadas. Los directivos tenían un nivel de entre 1 a 32 años en el puesto en que en ese momento se encontraban.

\section{Tipo de investigación}

Se trata de un estudio expost-facto transversal, que consiste en una medida única sin manipulación de las variables por parte del investigador (Hernández et al., 2014).

\section{Procedimiento}

La aplicación del instrumento se efectuó vía electrónica por medio de la plataforma en línea "Typeform". Se contactó a los participantes vía correo electrónico. Una vez que el participante accedía a la liga para contestar el cuestionario, se le presentó un consentimiento informado para autorizar el uso de la información proporcionada. También se le garantizó la confidencialidad de sus datos y se le aseguró que la medición no era una evaluación de desempeño. Se requirió de un tiempo promedio de 10 minutos para contestar la escala. Una vez que el participante terminaba de contestar, se le agradecía su participación y se cerraba la ventana del cuestionario. La aplicación de la escala a la muestra total tuvo una duración de dos meses. 


\section{Mediciones}

La escala de toma de decisiones en directivos (ETDD) parte de la lógica propuesta por Siebert y Kunz (2016) y retoma la lógica de las dimensiones enfocadas en habilidades, así como algunos de los ítems de la escala. La versión final se conformó de acuerdo con las siguientes dimensiones planteadas en el instrumento original: 1) identificación de objetivos; 2) identificación de alternativas; 3) identificación de información, y 4) uso de radar decisional. Durante el proceso de traducción, y con el objetivo de una mejor comprensión en el contexto del habla hispana, se modificó el nombre de la cuarta dimensión para la ETDD, cambiando "Uso de radar decisional" por "Análisis de las consecuencias". Por otro lado, se generaron más ítems que hacían referencia a conductas específicas de los directivos, complementando cada una de las dimensiones. La ETDD se constituyó por 21 ítems divididos en las siguientes cuatro dimensiones: 1) identificación objetivos (seis ítems); 2) identificación de alternativas (cinco ítems); 3) identificación de información (cuatro ítems), y 4) análisis de las consecuencias (seis ítems) (anexo 1). Se utilizó una escala tipo Likert con cinco opciones de respuesta en términos de frecuencia (1 "nunca", 2 "rara vez", 3 "a veces", 4 "casi siempre", y 5 "siempre"). Un trabajo previo destacó la validez de contenido de la escala (Ruiz y Vega, 2018) (anexo 1).

\section{ANÁlisis ESTAdísticos}

Para efectuar los análisis estadísticos se utilizaron los programas Factor versión 10.8.03, SPSS y AMOS versión 22. El orden de los análisis hechos fue el diagnóstico de normalidad en los puntajes generales del instrumento y la normalidad multivariada en los ítems. Para complementar el análisis se obtuvieron estadísticos descriptivos y se siguieron los criterios recomendados por Lloret-Segura, Ferreres-Traver, Hernández-Baeza y Tomás-Marco (2014) y Quero (2010).

Para evaluar la adecuación de los ítems al análisis factorial se consideraron los criterios recomendados por Ferrando y Anguiano-Carrasco (2010) y Pérez y Medrano (2010). Estos criterios consideran la normalidad multivariante, el estadístico Kaiser-Mayer-Olkin (KMO), la prueba de esfericidad de Bartlett y valor de determinante diferente de 0 . El análisis factorial exploratorio se hizo con un procedimiento de análisis robusto con muestreo simulado (bootstraping) siguiendo las recomendaciones propuestas por Lloret-Segura et al. (2014), Efron (1987), Cupani, Vaiman, Font, Pizzichini y Saretti (2012) y Lambert, Wildt y Durand (1991). Por último, se evaluó la consistencia interna de las dimensiones con el estadístico Omega propuesto por McDonald (1999).

\section{Resultados}

En la tabla 1 se muestran los estadísticos descriptivos y las pruebas de normalidad para el puntaje total del instrumento. Se aprecia la presencia de un sesgo negativo en la distribución que afecta la concentración de los valores en el punto modal de la distribución. Sin embargo, el análisis de normalidad con la prueba Shapiro Wilk reveló evidencia de normalidad.

\section{Tabla 1.}

Estadísticos descriptivos y pruebas de normalidad del puntaje total.*

\begin{tabular}{|c|c|c|c|c|c|c|}
\hline$M(S)$ & $\begin{array}{l}95 \% \\
\text { IC }\end{array}$ & MDN & $\begin{array}{c}\text { RANGO } \\
\text { NTERCUARTIL }\end{array}$ & Asımetría & Curtosis & $\begin{array}{l}\text { EVALUACIÓN } \\
\text { NORMALIDAD }\end{array}$ \\
\hline $\begin{array}{l}74.13 \\
(9.31)\end{array}$ & $\begin{array}{l}\text { [72.93, } \\
75.33]\end{array}$ & 74 & 11 & -0.285 & -0.096 & $\begin{array}{c}W(235)= \\
0.989 p= \\
0.068\end{array}$ \\
\hline
\end{tabular}

En la figura 1 se muestra un histograma y una gráfica cuantil-cuantil (Q-Q) para evaluar la normalidad de la distribución de los puntajes totales del instrumento. Primero, la distribución de puntuaciones totales presenta un sesgo negativo sin que esto aleje mucho su forma funcional a una curva normal. De modo adicional, los puntos en el gráfico Q-Q no muestran un alejamiento pronunciado a la línea recta ni una forma sigmoidal pronunciada. Estos resultados, junto con la prueba de normalidad en la tabla 1, proporcionan evidencia de normalidad en los puntajes generales.
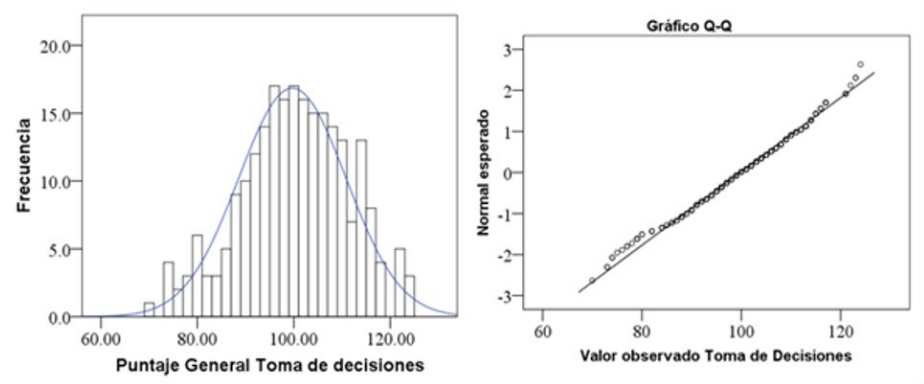

Figura 1.

Análisis de la distribución de los puntajes totales. El panel izquierdo es un histograma y el panel derecho un gráfico cuantil-cuantil $(Q-Q)$.

Después se hizo una prueba de normalidad multivariante con la prueba de Mardia para curtosis y asimetría (Mardia, 1970). Los resultados para curtosis b $1 p$ $=25.48 \mathrm{k}=0.758 \mathrm{p}=0.44$ y asimetría $\mathrm{b} 2 \mathrm{p}=2.89 \mathrm{k}=$ 
Tabla 2.

Estadísticos descriptivos de los ítems.

\begin{tabular}{|cccccccccc}
\hline Ítem & $\mathbf{M}$ & $\mathbf{S}$ & $\mathbf{R}$ & AsimetríA & Ítem & $\mathbf{X}$ & $\mathbf{S}$ & $\mathbf{R}$ & AsimetríA \\
\hline 1 & 4.472 & 0.674 & 0.596 & -1.245 & 12 & 4.566 & 0.619 & 0.552 & -1.233 \\
\hline 2 & 4.497 & 0.643 & 0.592 & -.916 & $13^{*}$ & 3.642 & 1.247 & 0.310 & -.709 \\
\hline 3 & 4.455 & 0.716 & 0.638 & -1.140 & 14 & 4.510 & 0.712 & 0.415 & -1.684 \\
\hline 4 & 4.502 & 0.681 & 0.602 & -1.191 & 15 & 4.408 & 0.786 & 0.568 & -1.501 \\
\hline 5 & 4.445 & 0.722 & 0.683 & -1.280 & $16^{*}$ & 3.812 & 1.108 & 0.445 & 7023 \\
\hline 6 & 4.561 & 0.633 & 0.517 & -1.252 & 17 & 4.387 & 0.794 & 0.518 & -1.428 \\
\hline $7 *$ & 3.080 & 1.280 & 0.261 & -.115 & $18^{*}$ & 3.829 & 1.167 & 0.410 & -.768 \\
\hline 8 & 4.297 & 0.748 & 0.676 & -.734 & 19 & 4.421 & 0.677 & 0.635 & -.835 \\
\hline 9 & 4.391 & 0.745 & 0.567 & -1.403 & $20^{*}$ & 4.178 & 1.017 & 0.466 & -1.321 \\
\hline 10 & 4.472 & 0.681 & 0.619 & -1.004 & 21 & 4.255 & 0.833 & 0.468 & -1.089 \\
\hline $11^{*}$ & 2.838 & 1.226 & 0.229 & .116 & & & & & \\
\hline *ítem invertidos. & & & & & & & \\
\hline
\end{tabular}

$24.15 \mathrm{p}=0.23$ demostraron evidencia de normalidad multivariante. En la tabla 2 se muestran los estadísticos descriptivos de los ítems. Se decidió eliminar todos los ítems que tuvieran correlaciones ítem total negativas o inferiores a 0.30, así como valores de asimetría superiores al valor absoluto de dos, como lo sugieren Lloret-Segura et al. (2014), Cozby (2005) y Quero (2010). Los ítems eliminados fueron el 7 y el 11 por no tener correlaciones ítem totales al menos de 0.30 .

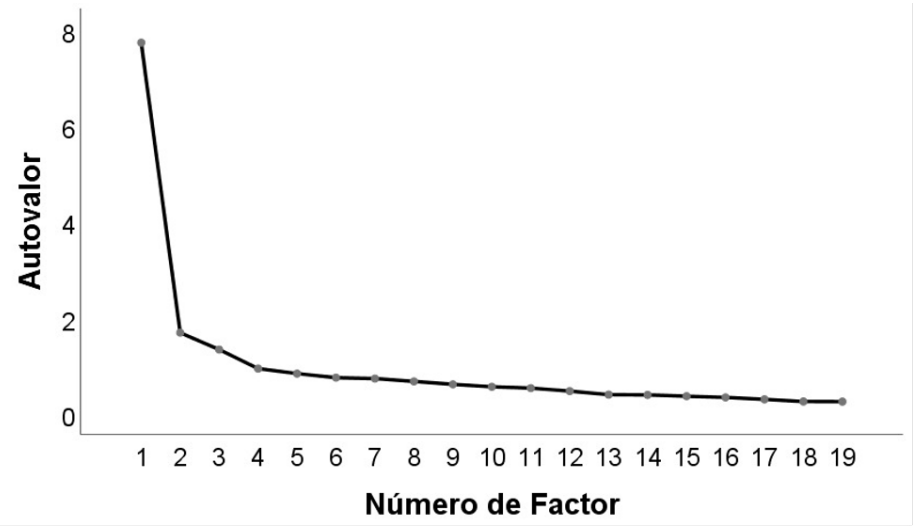

Figura 2.

Gráfico de sedimentación para determinar la cantidad de dimensiones tomando como criterio el punto de inflexión; se observan tres posibles factores.

Después de eliminar los ítems de acuerdo con los criterios indicados, se hizo la valoración de la adecuación de los datos al análisis factorial exploratorio (AFE) como mencionan Ferrando y Anguiano-Carrasco (2010) y Pérez y Medrano (2010). Se consideraron como valo- res aceptables: 1) valores del índice Kaiser-Mayer-Olkin $(\mathrm{KMO})$ superior a $0.80 ; 2)$ una prueba de esfericidad de Bartlett con un valor de significancia inferior a 0.05, y 3) un valor del determinante diferente de 0 . En este caso se obtuvieron valores aceptables en el coeficiente $\mathrm{KMO}=0.921$ y la prueba de esfericidad de Bartlett X2 = 2019.307 (153), $p<0.001$ y un valor del determinante diferente de 0 (0.00061).

En la figura 2 se muestran los autovalores como función del número de factores. En el gráfico se identificó por el punto de inflexión la posibilidad de tres factores.

Para el análisis factorial se utilizó el método de extracción de máxima verosimilitud porque se demostró normalidad multivariante y una rotación oblimin directa, ya que se asumió correlación entre factores (Lloret-Segura et al., 2014). Para evitar estimaciones sesgadas, se utilizó un análisis robusto para la estimación de las cargas factoriales a partir del método de muestreo Monte Carlo con 500 muestras seleccionadas a partir del método de percentil por sesgo corregido y acelerado propuesto por Efron (1987) con un intervalo de confianza de $95 \%$ en las cargas factoriales. Al hacer el análisis factorial se considera una estructura aceptable como aquella que cumpla con los siguientes criterios: la carga factorial mínima para considerar la posible pertenencia de un ítem respecto a alguna dimensión fueron de 0.25 en el intervalo inferior; cada ítem sólo puede pertenecer a una dimensión, por lo que los ítems con saturaciones secundarias con cargas que difieran al menos en una décima fueron excluidos del análisis, y cada posible factor está constituido por al menos tres ítems con una clara 
relación semántica entre cada uno de ellos (Cupani et al., 2012; Lambert, Wildt y Durand, 1991).

En la tabla 3 se muestra la matriz factorial con las cargas para cada ítem. Durante el análisis se eliminaron los ítems 6, 12, 19, 20 y 21 por presentar cargas factoriales menores al criterio de 0.25 en el intervalo inferior, quedando una solución con tres factores y 14 ítems. y explica $43.5 \%$ de la varianza, con $\Omega=0.91$; el segundo factor se identificó como "Identificación de objetivos organizacionales", con $11.05 \%$ de la varianza explicada y $\Omega=$ 0.68 ; mientras que en el tercer factor se agruparon ítems que correspondían a "Decisiones precipitadas", explicando $8.7 \%$ de varianza con $\Omega=0.63$. El omega general resultó en 0.92. Los ítems finales se muestran en el anexo 2.

Tabla 3

Matriz factorial con los ítems restantes

\begin{tabular}{|cccccccccc|}
\hline ÍteM & F1 & \multicolumn{2}{c}{ Cl $95 \%$} & F2 & \multicolumn{2}{c}{ C195\% } & \multicolumn{2}{c|}{ F3 } & \multicolumn{2}{c|}{ CI95\% } \\
\hline & & INF & SUP & & INF & SUP & 3 & INF & SUP \\
\hline 1 & .677 & .505 & .822 & .044 & -.170 & .213 & .039 & -.125 & .235 \\
\hline 2 & .888 & .799 & .999 & -.101 & -.234 & .009 & -.052 & -.155 & .032 \\
\hline 3 & .767 & .622 & .874 & .020 & -.117 & .139 & .043 & -.072 & .166 \\
\hline 4 & .829 & .700 & .958 & -.085 & -.230 & .050 & .004 & -.099 & .124 \\
\hline 5 & .720 & .431 & .836 & .054 & -.081 & .158 & .106 & -.016 & .276 \\
\hline 8 & .580 & .429 & .729 & .230 & .059 & .385 & .091 & -.057 & .233 \\
\hline 9 & .578 & .397 & .731 & .245 & .074 & .428 & -.086 & -.230 & .040 \\
\hline 10 & .638 & .473 & .764 & .137 & .004 & .298 & -.022 & -.157 & .085 \\
\hline 13 & -.005 & -.145 & .145 & -.014 & -.125 & .142 & .463 & .251 & .657 \\
\hline 14 & -.040 & -.215 & .095 & .814 & .627 & 1.035 & -.055 & -.144 & .047 \\
\hline 15 & .064 & -.100 & .222 & .706 & .516 & .889 & .139 & .047 & .246 \\
\hline 16 & -.040 & -.168 & .099 & .050 & -.075 & .164 & .698 & .408 & .992 \\
\hline 17 & .202 & .040 & .389 & .577 & .337 & .770 & -.034 & -.0174 & .087 \\
\hline 18 & .156 & .029 & .323 & -.090 & -.227 & .051 & .535 & .274 & .757 \\
\hline
\end{tabular}

El ajuste del análisis factorial exploratorio se evaluó con base en los criterios sugeridos por Schumacker y Lomax (2010), basados en los indicadores de bondad de ajuste absoluto, de ajuste incremental y parsimonia. En la tabla 4 se muestran los índices de ajuste absoluto del modelo. De acuerdo con estos índices es evidente que hay una similitud entre el modelo empírico y teórico, chi cuadrada no significativos y el RMSEA con un valor inferior a 0.05. Los índices de bondad de ajuste robustos indican una buena adecuación de la estructura factorial. Por otro lado, los índices de ajuste incremental indican un buen ajuste con valores mayores a 0.95 en los índices NNFI y CFI. Por último, el índice de parsimonia resultó menor a 3, lo que se interpreta como un buen ajuste.

El resultado final del análisis presenta una solución de tres factores que hacen referencia a las acciones que el directivo considera o no para tomar una decisión dentro de su ámbito laboral. El primer factor hace referencia a "Identificación y análisis de información y alternativas"

\section{Discusión}

La escala de toma de decisiones directivas (ETDD) mostró adecuados índices de confiabilidad en general y por cada una de las tres dimensiones presentadas en el modelo. Esto supone que el instrumento podrá arrojar datos consistentes de las habilidades que competen a la toma de decisiones al ser aplicado en muestras similares, permitiendo un diagnóstico oportuno de necesidades de capacitación específica en directivos.

Los procedimientos de validez estadística efectuados mostraron que los ítems no se agruparon en las dimensiones propuestas por Siebert y Kunz (2016). Dichas dimensiones eran 1) identificación sistemática de objetivos; 2) identificación sistemática de alternativas; 3 ) búsqueda sistemática de información, y 4) análisis de las consecuencias. Sin embargo, nuestro análisis reveló una solución diferente para los ítems basada en tres dimensiones. La agrupación de los ítems tiene consistencia teórica con la propuesta. 
Tabla 4.

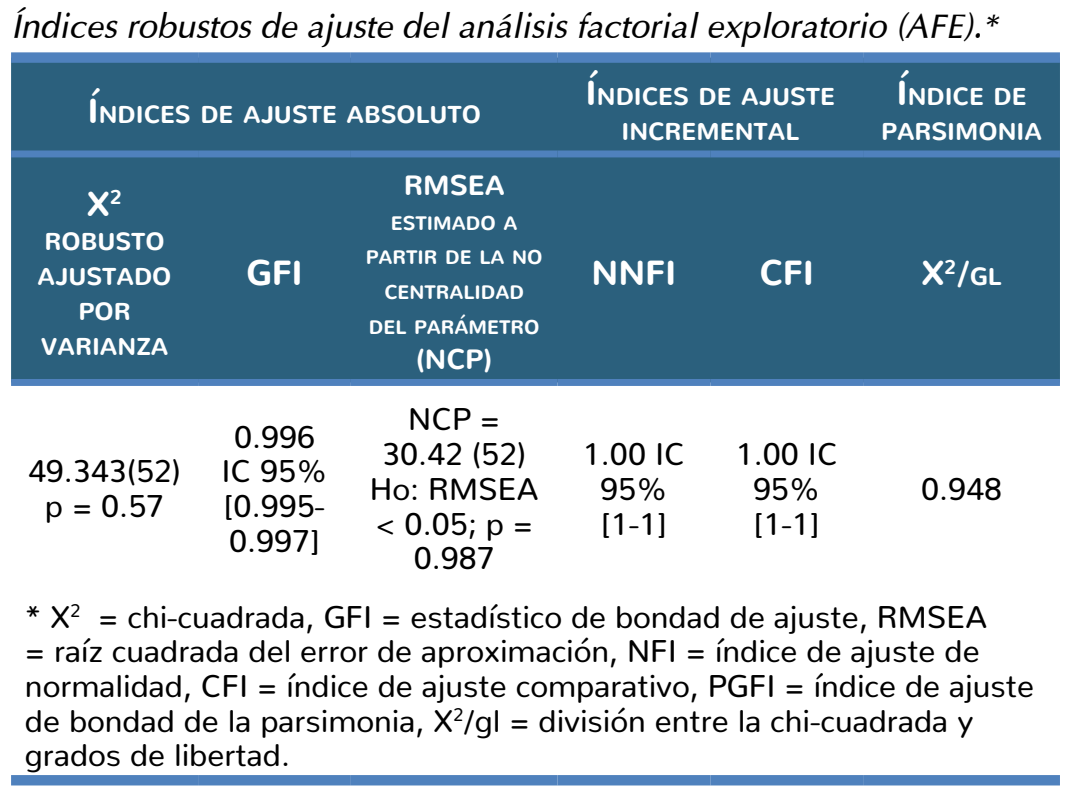

Debido a lo anterior, las tres dimensiones obtenidas fueron denominadas: 1) identificación y análisis de información y alternativas; 2) identificación de objetivos organizacionales, y 3) toma de decisiones precipitadas.

En el modelo obtenido en este estudio, la primera dimensión hace referencia a la habilidad de que antes de tomar una decisión los directivos recuperen información que les permita tener un panorama más amplio para elegir el mejor curso de acción. Esto lo hacen identificando las posibles consecuencias de sus acciones y los riesgos implicados, así como cada una de las opciones que pueden seleccionar para tomar una decisión satisfactoria. Lo anterior está de acuerdo con lo hallado en la literatura en cuanto a la generación de alternativas previa a la toma de decisiones y a la anticipación de las consecuencias (Cabeza de Vergara, Muñoz y Vivero, 2004; Kepner y Tregoe, 1972; Peñaloza, 2010; Solano, 2003; Vélez, 2006).

Por otro lado, la segunda dimensión se refiere a la habilidad de que, al tomar una decisión, los directivos se enfocan en los objetivos y necesidades de la organización, las necesidades de su lugar de trabajo, los objetivos emergentes y la forma en que es necesario que se tome la decisión para cumplir con ellos. Este resultado se debe a la manera en que se agruparon los ítems en esta dimensión, aunque también es similar a los reportados en la literatura, en donde se ha venido indicando que los directivos basan sus decisiones en las necesidades, objetivos y requerimientos de la organización, con el objetivo de que se ésta se mantenga a la vanguardia (Martínez, Bonilla y Botero, 2014; Cabeza de Vergara y Muñoz, 2006; Solano, 2003; Vélez, 2006).
Por último, la tercera dimensión hace referencia a que algunos directivos suelen tomar decisiones importantes sin recurrir o enfocarse de modo parcial en la información que se tiene de la situación. Estos directivos no identifican las consecuencias a corto y largo plazos o se enfocan sólo en una alternativa. Lo anterior parece insertarse en lo indicado por la literatura cuando se dice que, en ocasiones, los directivos otorgan poca importancia a la utilización de ayudas informativas en el proceso de toma de decisiones (Cabeza de Vergara y Muñoz, 2006). Dicha poca importancia se debe a factores como la presión del tiempo para decidir rápidamente, la carga de ofrecer resultados efectivos a corto plazo, o a que en varias ocasiones los datos e información que se tienen para tomar una decisión son muy numerosos y difíciles de ordenar (Citroen, 2011). A pesar de lo anterior, el limitado número de ítems en este factor, así como el valor de consistencia interna y las saturaciones obtenidas, indican que la presencia de este factor debe tomarse con cautela. También indica la necesidad de investigaciones posteriores donde se pueda replicar la misma estructura con este factor porque, si bien cuenta con tres ítems, la confiabilidad es muy baja (Lloret-Segura et al., 2014).

De manera general, los resultados de este trabajo contribuyen a la generación de un instrumento que explique la toma de decisiones a partir de habilidades específicas en las organizaciones. Esto, en contraste con otros instrumentos que se enfocaban en la medición de aspectos genéricos como los estilos decisionales intuitivo, racional y ante el conflicto, los rasgos de personalidad de los individuos al tomar decisiones o la valoración positiva o negativa respecto a las consecuencias futuras (Azzollini 
y Depaula, 2013; Mann et al., 1997; Nenkov, Inman y Hulland, 2008; Sjöberg, 2003; Maciá et al., 1996). Hacer la medición de la toma de decisiones con este nuevo instrumento podría permitir un diagnóstico más preciso de las áreas de oportunidad, abriendo la posibilidad de generar entrenamientos en las habilidades específicas que los directivos deben ejecutar al tomar una decisión efectiva (Ruiz y Vega, 2017; VandenBos, 2007).

\section{Conclusiones}

Este estudio ofrece un panorama diferente a la medición de la toma de decisiones de directivos dentro de las organizaciones, caracterizándola en tres componentes diferentes que no necesariamente suceden en secuencia. El instrumento aquí propuesto resulta una aportación al campo del análisis del comportamiento directivo porque ahora se cuenta con un instrumento que permita caracterizar la toma de decisiones en el ámbito laboral y enfocado en directivos. Esto permite: 1) realizar investigación mostrando evidencia de las habilidades de los directivos relativas a la toma de decisiones, y 2) contar con un instrumento que permita efectuar un diagnóstico para identificar las áreas de oportunidad de un directivo en su toma de decisiones cotidianas. De lo anterior, también se desprendería la posibilidad de generar entrenamientos específicos con base en el diagnóstico, dejando de lado aspectos como estilos decisorios o rasgos de personalidad. Ruiz y Vega (2017) detallan cómo desarrollar una capacitación específica de este tipo. Estos autores exponen que el primer paso es definir las variables de interés. Después se hace una evaluación a partir de instrumentos o entrevistas que permitan elaborar un diagnóstico. Una vez hecho esto, se procede a usar la lógica de los diseños intrasujeto para aplicar técnicas de modificación conductual pertinentes a la situación de análisis. Al final se podría efectuar un seguimiento para identificar si los cambios observados en la intervención se mantienen.

\section{SUGERENCIAS}

Una limitación importante del estudio fue que sólo se proporcionó evidencia de un número reducido de fuentes que suman a la validez de la medición. En este sentido, las líneas de trabajo a seguir se relacionarían con garantizar validez convergente y discriminante de la toma de decisiones utilizando otras variables que compartan la lógica teórica con que se desarrolló este instrumento.

Por otro lado, una manera de aportar validez predictiva a la concepción de la toma de decisiones como conductas explícitas sería mediante el diseño de intervenciones con directivos enfocadas en mejorar su proceso de toma de decisiones. Dichos esfuerzos podrían seguir el uso de metodología intrasujeto para verificar 1) si se puede modificar el proceso en cada individuo, y 2) si los resultados presentan una covariación positiva con indicadores relacionados con el éxito organizacional.

\section{Referencias}

Analoui, F., Labbaf, H., \& Noorbakhsh, F. (2000). Identification of clusters of managerial skills for increased effectiveness. The case of the steel industry in Iran. International Journal of Training and Development, 4 (3), 217-234. doi: 10.1111/1468-2419.00109

Anderson, D. R., Sweeney, D. J., Williams, T. A., Camm, J. D., Cochran, J.J., Fry, M. J., \& Ohlmann, J. W. (2016). Métodos cuantitativos para los negocios. Recuperado de https:// issuu.com/cengagelatam/docs/m todos cuantitativos para los neg

Azzollini, S. C., \& Depaula, P. D. (2013). Análisis de validez y confiabilidad de una escala para la evaluación de estilos decisorios operativos. EVALUAR, 13(1), 38-60. doi: https:// doi.org/10.35670/1667-4545.v13.n1.6795

Bruine de Bruin, W., Parker A. M., \& Fischhoff, B. (2012). Explaining Adult Age Differences in Decision-making Competence. Journal of Behavioral Decision Making, 25, 352-360. doi: doi:https://doi.org/10.1002/bdm.712

Butler, R. (1991). Designing Organizations. A Decision-Making Perspective. Londres: Routledge.

Cabeza de Vergara, L., \& Muñoz, S. A. (2006). Análisis del proceso de toma de decisiones en las grandes empresas de Barranquilla utilizando el análisis por conglomerados. Pensamiento y Gestión, (20), 55-109. Recuperado de http:// rcientificas.uninorte.edu.co/index.php/pensamiento/ article/view/3573

Cabeza de Vergara, L., Muñoz, S. A. E., \& Vivero, S. S. M. (2004). Aproximación al proceso de toma de decisiones en la empresa barranquillera. Pensamiento y Gestión. (17), 1-38. Recuperado de http://rcientificas.uninorte.edu.co/ index.php/pensamiento/article/view/3601

Citroen, C. L. (2011). The role of information in strategic decisionmaking. International Journal of Information Management, 31 (6), 493-501. doi: 10.1016/j.ijinfomgt.2011.02.0057

Cozby,P.C.(2005). Métodos de investigación del comportamiento (8th ed.). México: McGraw-Hill

Cupani, M., Vaiman, M., Font, M. L., Pizzichini, F., \& Saretti, B. (2012). Análisis factorial confirmatorio del NEO-FFI utilizando parcelización de ítems y método Bootstrap. Avaliação Psicológica, 11(2), 159-168. Recuperado el 13 de julio de 2020, de http://pepsic.bvsalud.org/scielo. php?script=sci arttext\&pid=S1677-04712012000200002

DeVellis, R. F. (2017). Scale Development: Theory and Applications (4th ed.). Thousand Oaks, CA: Sage

Efron, B. (1987). Better bootstrap confidence intervals (with discussion). Journal of the American Statistical Association, 82, doi:171-20. 10.2307/2289144 
Ferrando, P.J., \& Anguiano-Carrasco, C. (2010). El análisis factorial como técnica de investigación en Psicología. Papeles del psicólogo, 31 (1), 18-33. Recuperado de http:// www.redalyc.org/articulo.oa?id $=77812441003$

Freixa i B., E. (2003). ¿Qué es la conducta? International Journal of Clinical and Health Psychology, 3 (3), 595-613. Recuperado de http://www.redalyc.org/articulo.oa?id=33730310

Furby, L., \& Beyth-Marom, R. (1992). Risk taking in adolescence: A decision-making perspective. Developmental Review, 12 (1), 1-44. doi: https://doi.org/10.1016/02732297(92)90002-1

Griffin, R., \& Van Fleet, D. (2016). Habilidades directivas evaluación y desarrollo. México: CENGAGE Learning

Hernández S., R., Fernández-Collado, C. \& Baptista, P. (2014). Metodología de la investigación. México: Mc Graw Hill.

Judge, T. A., Bono, J. E., llies, R., \& Gerhardt, M. W. (2002). Personality and leadership: A qualitative and quantitative review. Journal of Applied Psychology, 87(4), 765-780. doi: https://doi.org/10.1037/0021-9010.87.4.765

Kepner, C. H., \& Tregoe, B. B. (1972). El directivo racional: Enfoque sistemático a la resolución de problemas y la toma de decisiones. México: McGraw-Hill

Kyriazos, T. A, \& Stalikas, A. (2018). Applied Psychometrics: The Steps of Scale Development and Standardization Process. Psychology, 9, 2531-2560. doi: 10.4236/ psych.2018.911145

Lambert, Z. V., Wildt, A. R., \& Durand, R. M. (1991). Approximating Confidence Intervals for Factor Loadings. Multivariate Behavioral Research, 26(3), 421-434. doi: 10.1207/ s15327906mbr2603_3.

Lloret-Segura, S. Ferreres-Traver, A. Hernández-Baeza, A., \& Tomás-Marco, I. (2014). El análisis factorial exploratorio de los ítems: una guía práctica, revisada y actualizada. Anales de psicología, 30 (3), 1151-1169. doi: http://dx.doi. org/10.6018/analesps.30.3.199361

Maciá, A., Barbero, I., Pérez-Llantada, C., Vila, E., Navas M. J., \& Mandakovic, T. (1996). Estudio interno de una escala de toma de decisión: E.T.D. Revista de Psicología General y Aplicada. 49,2, 267-278. Recuperado de https://www. researchgate.net/publication/28175031

Madrigal, T.B.E. (2009). Habilidades directivas. México: McGraw Hill

Mann, L., Burnett, P., Radford, M., \& Ford, S. (1997). The Melbourne Decision Making Questionnaire: An Instrument for Measuring Patterns for Coping with Decisional Conflict. Journal of Behavioral Decision Making, 10 (1), 1-19. doi: https://doi.org/10.1002/(SICI)10990771(199703)10:1<1::AID-BDM242>3.0.CO;2-X

Mardia, K.V. (1970). Measures of Multivariate Skewness and Kurtosis with Applications Measures of Multivariate Skewness and Kurtosis with Applications. Biometrika, 57(3), 519. doi:10.2307/2334770.

Martínez, P. G. D., Bonilla, B. L. F., \& Botero, O. B. G. (2014). El procesamiento de la información y el carácter racional de la toma de decisión en el ámbito organizacional. Importancia e implicaciones. Gestión y desarrollo, 11 (1), 191-203. doi:https://doi.org/10.21500/01235834.2128

Moreno-Jiménez, B., Blanco-Donoso, L., Aguirre- Camacho, A., De Riva, S., \& Herrero, M. (2014). Habilidades sociales para las nuevas organizaciones. Behavioral Psychology
/ Psicología Conductual, 22, 585-602. Recuperado de https://www.researchgate.net/publication/271507225 Habilidades Sociales para las Nuevas Organizaciones

McDonald, R.P. (1999). Test theory: A unified treatment. Mahwah, NJ: Lawrence Erlbaum

Nenkov, G.Y., Inman, J.J., \& Hulland, J. (2008). Considering the Future: The Conceptualization and Measurement of Elaboration on Potencial Outcomes. Journal of Consumer Research, 35 (1), 126-141. doi:10.1086/525504

Peñaloza, P. M. (2010). Teoría de las decisiones. Perspectivas, (25), 227-240. Recuperado de http://www.redalyc.org/ articulo.oa?id $=425942454012$

Pereda, J., López, T., \& González, F. (2014). Las habilidades directivas como ventaja competitiva. El caso del sector público de la provincia de Córdoba (España). Intangible Capital, 10(3), 528-561. Recuperado de http://www. intangiblecapital.org

Pérez G.G., Barroso T. F. G., Escalante F. J. J., \& Eliseo D. H. (2008). Medición de habilidades directivas en los institutos tecnológicos del sureste de México. Avance de Investigación. (5), 193-234.

Pérez, E., \& Medrano, L. (2010). Análisis factorial exploratorio: Bases conceptuales y metodológicas. Revista Argentina de Ciencias del Comportamiento, 2 (1), 5866. Recuperado de https://dialnet.unirioja.es/servlet/ articulo?codigo $=3161108$

Quero, V. M. (2010). Confiabilidad y coeficiente Alpha de Cronbach. Telos, 12 (2), 248-252. Recuperado de http:// www.redalyc.org/articulo.oa?id $=99315569010$

Quintero, P. F, Granada, K., Álvarez, C.T., \& Calderón, G. (2011). El director como líder creativo. AD-minister. 19, 6781. Recuperado de http://www.redalyc.org/articulo. oa?id=322327250004

Ramón, D. I., Ollage V., J., Granda B., J., \& Naranjo S., C. (2017). Valoración de habilidades gerenciales personales a empleados de organizaciones públicas y privadas, por parte de directivos. INNOVA Research Journal, 2(8.1), 4255. doi: https://doi.org/10.33890/innova.v2.n8.1.2017.329

Rodríguez C., M., \& Posadas D., A. (2005). La Psicología en el escenario del trabajo: Una revisión. Revista Electrónica de Psicología Iztacala, 8(2), 60-80.

Ruíz, M. D., \& Vega, V. C. Z. (2017). Capacitación basada en evidencias. Una aportación tecnológica desde la psicología. Revista Iberoamericana de Psicología, 10 (2), 135-143. Recuperado de https://revistas.iberoamericana. edu.co/index.php/ripsicologia/article/view/12477

Ruíz, M. D. \& Vega, V. C. Z. (octubre, 2018). Caracterizando habilidades directivas: Una aproximación experimental a la toma de decisiones. En C. Juárez (presidencia), XXVIII Congreso Mexicano de Análisis de la Conducta. Congreso llevado a cabo en Boca del Río, Veracruz, México.

Rynes, S.L., Colbert, A. E., \& Brown, K. G. (2002). HR Professionals' beliefs about effective human resource practices: correspondence between research and practice. Human Resource Management, 41 (2), 149-174. doi: https://doi. org/10.1002/hrm.10029

Salazar, D. (2017). La gestión del tiempo como factor clave en las habilidades directivas aplicadas al sector turístico. Gran Tour, (15), 26-42. 
Salvador, M., Fuente, M. \& Álvarez, J. (2009). Las habilidades sociales en directores de centros escolares. European Journal of Education and Psychology, 2(3), 275-288. Recuperado de http://www.redalyc.org/articulo. oa?id=129312574008

Sánchez, J., Aguirre, M., Barrales, A., Vergara, J., \& Mota, I. (2015). Las habilidades directivas. En Red Mexicana de Investigadores en Estudios Organizacionales (ed.), El análisis organizacional en México y América Latina, Retos y perspectivas a 20 años de estudios (pp. 125164). México: Hess. Recuperado de remineo.org/images/ documentos/memorias/ciao/xiiiciao/aomal.pdf

Schumacker, R. E., \& Lomax, R. G. (2010). A Biginner's Guide to Structural equation modeling. New York: Routledge.

Siebert, J., \& Kunz, R. (2016). Developing and validating the multidimensional proactive decision-making scale. European Journal of Operational Research, 249 (3), 864877. doi: https://doi.org/10.1016/j.ejor.2015.06.066

Sjöberg, L. (2003). Intuitive vs. Analytical decision making: which is preferred? Scandinavian Journal of Management, 19 (1), 17-29. doi: https://doi.org/10.1016/S0956-5221(01)00041-0

Solano, A. I. (2003). Toma de decisiones gerenciales. Tecnología en marcha, 16 (3), 44-51. Recuperado de https://www.academia.edu/35156699/Dialnet TomaDeDecisionesGerenciales

VandenBos, G. R. (ed.) (2007). APA dictionary of psychology. EUA: American Psychological Association.
Vega-Valero, C., Hernández-Toledano, R., García-Arreola, O., Nava-Quiroz, C., \& Ruíz-Méndez, D. (2019). Escala de habilidades de solución de problemas en directivos: Desarrollo y validación. EVALUAR, 19(3), 68-85. Recuperado de https://revistas.unc.edu.ar/index.php/ revaluar

Vélez, E. M. I. (2006). El proceso de toma de decisiones como un espacio para el aprendizaje en las organizaciones. Revista Ciencias Estratégicas, 14 (16), 153-169. Recuperado de http://www.redalyc.org/articulo.oa?id=151320326003

Veloso-Besio, C., Cuadra-Peralta, A., Gil-Rodríguez, F., QuirozCornejo, Á., \& Meza-Castro, S. (2015). Capacitación en trabajadores: Impacto de un programa, basado en psicología positiva y habilidades sociales, en satisfacción vital, satisfacción laboral y clima organizacional. Interciencia, 40 (11), 736-743. Recuperado de http://www. redalyc.org/articulo.oa?id $=33942541002$

Vickers, C., Dowell, S., \& House, S. (2019). A Role-Playing Simulation Strategy to Develop Prioritization, Delegation, and Time Management Skills. Teaching and Learning in Nursing, 14(1), 7-8. doi: https://doi.org/10.1016/]. TELN.2018.08.002

Whetten, D. A., \& Cameron, K. S. (2011). Desarrollo de habilidades directivas. México: Pearson Educación

Winterfeldt, D. V., \& Edwards, W. (1986). Decision Analysis and Behavioral Research. Cambridge: Cambridge University Press 


\section{AneXos}

\section{Anexo 1. Ítems de las dimensiones originales del instrumento de Toma de Decisiones en Directivos}

A continuación, se presentan los 21 ítems por dimensión, la enumeración corresponde a la manera en que fueron presentados en su aplicación y análisis estadístico. Posteriormente se muestra los ítems con la nueva agrupación de las dimensiones.

Dimensión

Identificación objetivos

Identificación de alternativas

Identificación de información

Análisis de las consecuencias

\section{Í́TEM}

6. Mis objetivos son claros cuanto tengo que tomar una decisión en mí trabajo.

12. Cuando tengo que tomar una decisión en mi trabajo, pienso de manera clara en el objetivo que quiero alcanzar.

14. En decisiones importantes, reflexiono sobre lo que requiere la organización.

15. Identifico los objetivos de la organización para generar alternativas de solución a un problema.

17. Pienso detenidamente en la manera de alcanzar los objetivos de la organización.

20. Tomo decisiones sin generar un objetivo previo.

3.Analizo los posibles resultados al evaluar las alternativas.

5.Considero todas las alternativas posibles antes de decidir.

9.Considero todas las alternativas generadas.

11.Elaboro mis alternativas teniendo solo la información inmediata disponible.

16. Considero únicamente la información de una alternativa.

2. Recopilo ordenadamente información relevante para tomar una decisión.

4. Al tomar decisiones reúno toda la información posible.

10.verifico mi información para asegurarme que tengo los elementos adecuados antes de tomar una decisión.

18. Tomo decisiones importantes sin recurrir al uso de reportes escritos o documentos normativos.

1. Antes de tomar una decisión en mi trabajo considero todas las posibles consecuencias.

7.Para tomar una decisión me centro poco en las consecuencias a largo plazo.

8.Para tomar una decisión evalúo de manera cuidadosa los riesgos de cada resultado en mi trabajo.

13.Para tomar una decisión no pienso en las consecuencias a corto plazo.

19. Evalúo la importancia de las consecuencias de mis decisiones. 


\section{Anexo 2. Ítems de las dimensiones resultantes del instrumento Toma de Decisiones en Directivos}

A continuación, se presentan los 15 ítems ordenados a partir de la agrupación de las dimensiones resultantes.

\section{Dimensión}

Identificación y análisis de información y alternativas

Identificación de objetivos organizacionales

Decisiones precipitadas

\section{ÍteM}

1. Antes de tomar una decisión en mi trabajo considero todas las posibles consecuencias.

2. Recopilo ordenadamente información relevante para tomar una decisión.

3.Analizo los posibles resultados al evaluar las alternativas.

4. Al tomar decisiones reúno toda la información posible.

5.Considero todas las alternativas posibles antes de decidir.

8.Para tomar una decisión evalúo de manera cuidadosa los riesgos de cada resultado en mi trabajo.

9.Considero todas las alternativas generadas.

10.verifico mi información para asegurarme que tengo los elementos adecuados antes de tomar una decisión.

12. Cuando tengo que tomar una decisión en mi trabajo, pienso de manera clara en el objetivo que quiero alcanzar.

14. En decisiones importantes, reflexiono sobre lo que requiere la organización.

15. Identifico los objetivos de la organización para generar alternativas de solución a un problema.

17. Pienso detenidamente en la manera de alcanzar los objetivos de la organización.

16. considero únicamente la información de una alternativa.

18. Tomo decisiones importantes sin recurrir al uso de reportes escritos o documentos normativos. 


\section{Meta-Análisis del Artículo}




\section{Dimensión Cuantitativa}

\section{Perfil de Evaluación entre pares}
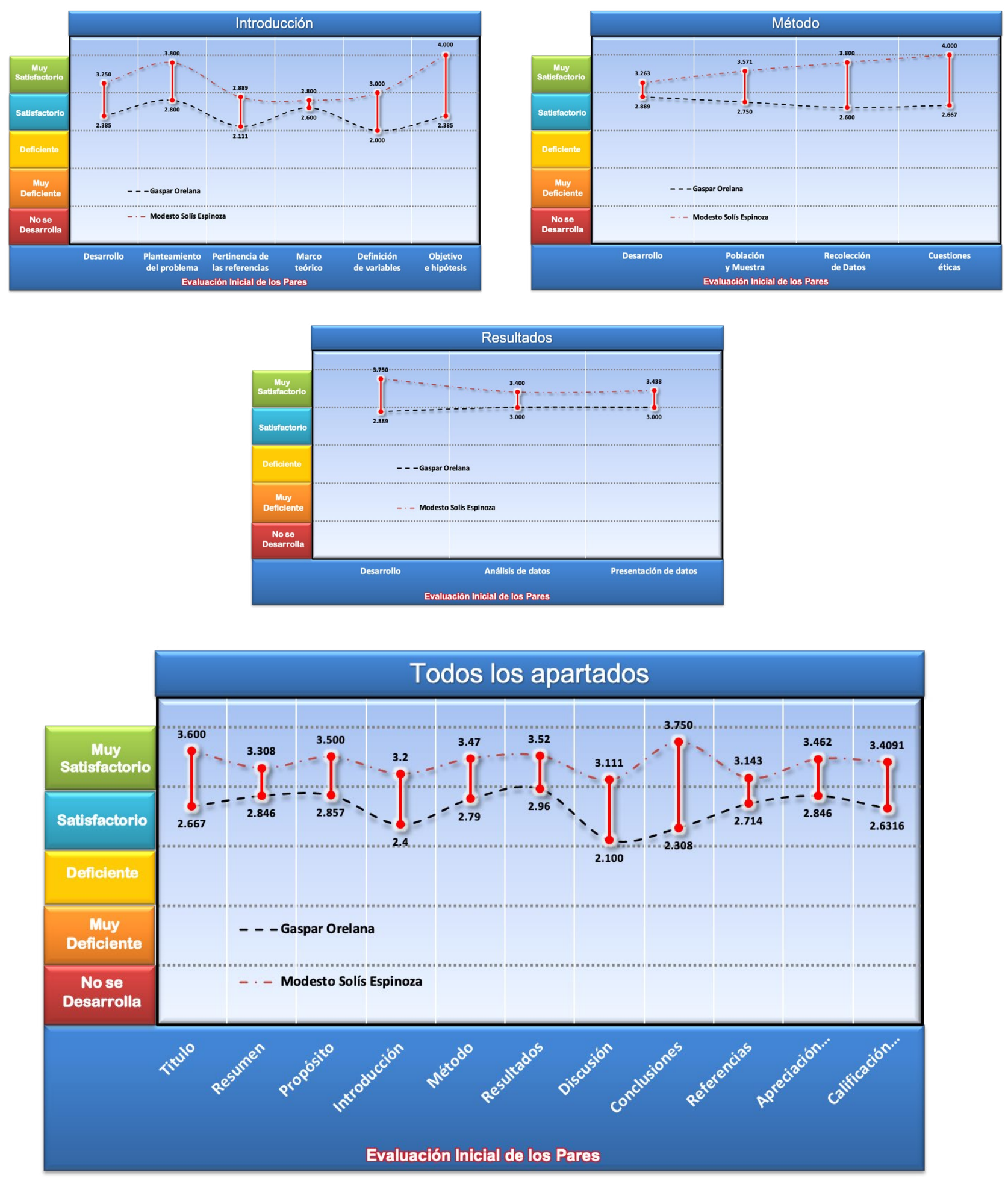


\section{Índice de Concordancia}

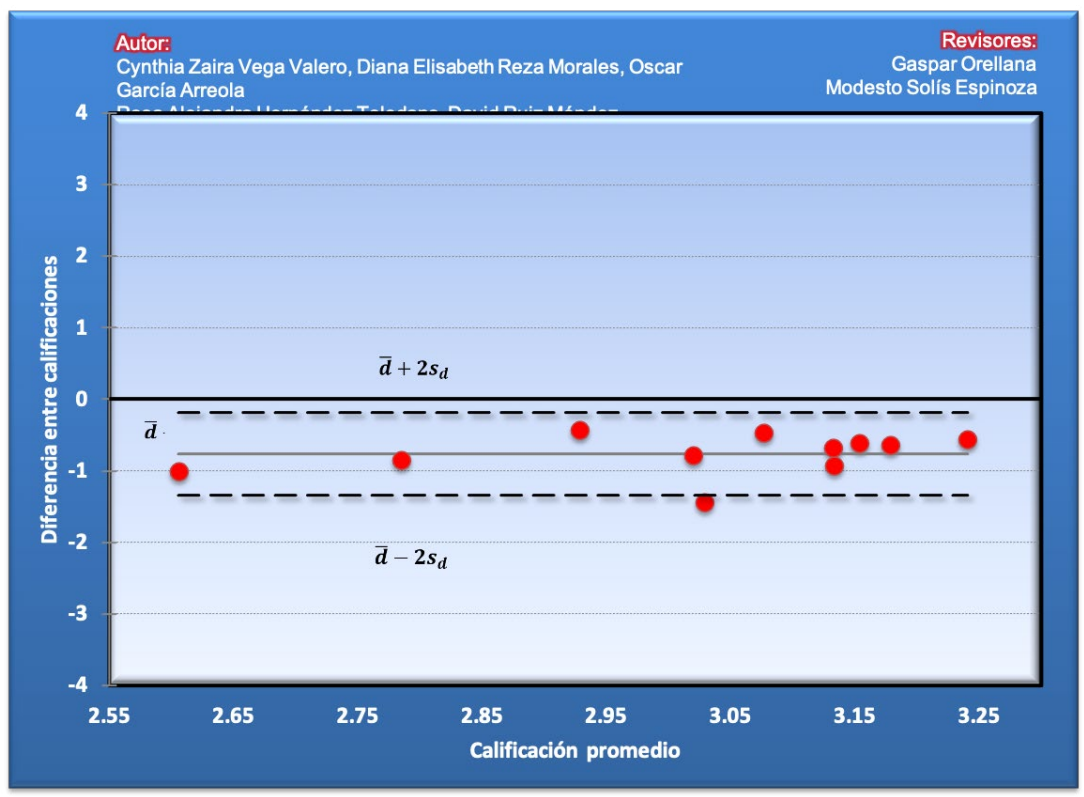

Índice de Acuerdo

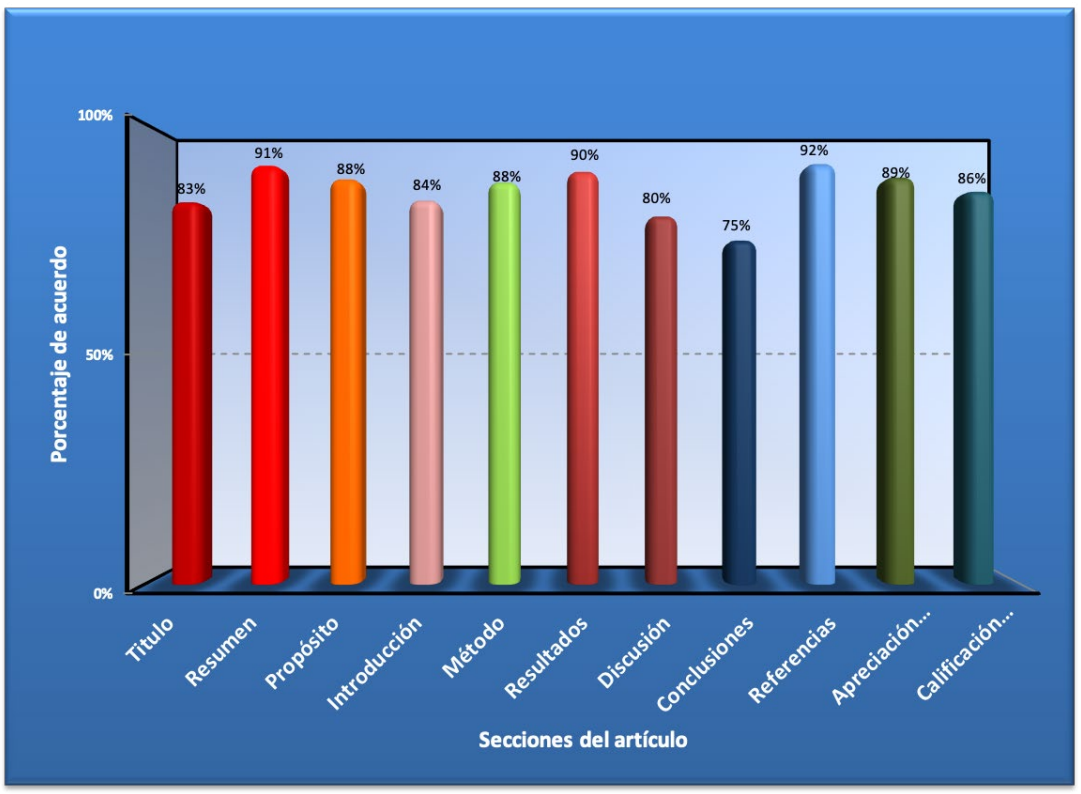




\begin{tabular}{|c|c|}
\hline Revisor 1 & Revisor 2 \\
\hline Gaspar Orellana & Modesto Solís Espinoza \\
\hline \multicolumn{2}{|c|}{ Título/Autoría } \\
\hline $\begin{array}{l}\text { la variable es escala de toma de decisiones el objetivo es } \\
\text { análisis psicométrico y propuesta para directivos de la es- } \\
\text { cala de otro autor }\end{array}$ & $\begin{array}{l}\text { Sugiero la modificación del título de manera que se } \\
\text { mencionen explícitamente las palabras "escala" ó "ins- } \\
\text { trumento", dado que por "estrategia de medición" se } \\
\text { podría entender que están incluidas otras actividades o } \\
\text { que la evaluación no consiste únicamente en el llenado } \\
\text { de una escala. Además de que así sería mucho más fácil } \\
\text { acceder al artículo en los buscadores cuando se haga } \\
\text { una búsqueda de instrumentos. }\end{array}$ \\
\hline \multicolumn{2}{|c|}{ Resumen } \\
\hline Omitir la importancia de estudio & $\begin{array}{l}\text { El resumen es claro pero mejoraría la redacción del párra- } \\
\text { fo introductorio, con lo que quedaría también más breve. }\end{array}$ \\
\hline \multicolumn{2}{|c|}{ Próposito del Estudio } \\
\hline Redactar la discusión en función de los objetivos & $\begin{array}{l}\text { El propósito queda claro y se guarda congruencia en las } \\
\text { diferentes partes del texto. }\end{array}$ \\
\hline
\end{tabular}




\begin{tabular}{|c|c|}
\hline Revisor 1 & Revisor 2 \\
\hline \multicolumn{2}{|c|}{ Introducción } \\
\hline $\begin{array}{l}\text { Agregar información teórica y antecedentes de psicome- } \\
\text { tría: validez, confiabilidad y análisis factorial }\end{array}$ & $\begin{array}{l}\text { Aunque queda clara la justificación de por qué estudiar } \\
\text { la toma de decisiones, podría ser útil complementar con } \\
\text { más información sobre por qué es clave, por qué darle } \\
\text { más importancia a esa habilidad por sobre las demás. Es } \\
\text { innegable que hay autores de hace muchos años que han } \\
\text { dado aportes que siguen vigentes hoy en día, sin embar- } \\
\text { go el texto contiene varias citas de hace más de } 20 \text { años, } \\
\text { por lo que sugeriría continuar la búsqueda de artículos } \\
\text { más recientes. Aunque se menciona la evaluación de } \\
\text { la toma de decisiones con base en los resultados, no se } \\
\text { ahonda en dicho aspecto, no queda claro si existen estra- } \\
\text { tegias para analizar los resultados o es meramente algo } \\
\text { que se deduce. Posteriormente mencionan el modelo } \\
\text { teórico de Toma de Decisiones Proactivas, sin embargo } \\
\text { no se explica dicho modelo o cómo es que se relacio- } \\
\text { nan sus dimensiones, aspectos relevantes al ser la base } \\
\text { del instrumento. Por otro lado, omitir a la personalidad } \\
\text { del la escala resulta contradictorio con el modelo teórico } \\
\text { de Siebert y Kunz, quienes en el desarrollo de su instru- } \\
\text { mento expusieron la relevancia de los rasgos de persona- } \\
\text { lidad (tomar iniciativa y deseo de mejora) como piezas } \\
\text { claves en la toma de decisiones, mi sugerencia es que se } \\
\text { justifique por qué no se incluyó dicho aspecto del mo- } \\
\text { delo teórico, o fundamentar explícitamente por qué no } \\
\text { es tan importante la personalidad de los directivos en la } \\
\text { toma de decisiones, de lo contrario incluirlo como una } \\
\text { limitación del estudio, dado que no están retomando el } \\
\text { modelo teórico como lo postularon los autores. }\end{array}$ \\
\hline \multicolumn{2}{|c|}{ Método } \\
\hline $\begin{array}{l}\text { Justificar mejor el uso de parámetros de algunos estadís- } \\
\text { ticos describir como se consiguieron los ítems de la va- } \\
\text { riable de estudio Señalar indicadores de cada dimensión } \\
\text { de la variable }\end{array}$ & $\begin{array}{l}\text { Al final del procedimiento la redacción no es clara, parece } \\
\text { que se repite parte del procedimiento cuando se mencio- } \\
\text { na la plataforma en línea. }\end{array}$ \\
\hline \multicolumn{2}{|c|}{ Resultados } \\
\hline Señalar autores que respalden las interpretaciones & $\begin{array}{l}\text { Se mencionan análisis factorial abierto y cerrado, sin em- } \\
\text { bargo la descripción de los mismos se semeja a los análisis } \\
\text { factorial exploratorio y confirmatorio, por lo que no me } \\
\text { queda claro el beneficio de usar esos términos más allá de } \\
\text { lo teórico. Sugiero que se modifique la Tabla } 4 \text { de forma } \\
\text { que sea más fácil su lectura, de modo que se pueda identi- } \\
\text { ficar más claramente las cargas de cada factor. }\end{array}$ \\
\hline
\end{tabular}




\begin{tabular}{|c|c|}
\hline Revisor 1 & Revisor 2 \\
\hline \multicolumn{2}{|c|}{ Discusión } \\
\hline $\begin{array}{l}\text { Agregar explicaciones psicométricas de los hallazgos, } \\
\text { refiriendo autores en los cuales se respalda }\end{array}$ & $\begin{array}{l}\text { Al final de la discusión se menciona que el proceso de } \\
\text { toma de decisiones puede no ser lineal, sin embargo no } \\
\text { se fundamenta o no queda claro el por qué de esa afirma- } \\
\text { ción, sería necesario hacerlo explícito. }\end{array}$ \\
\hline \multicolumn{2}{|c|}{ Conclusiones } \\
\hline Ceñirse a los objetivos y lo hallado & $\begin{array}{l}\text { Se menciona que se podrían hacer entrenamiento dejan- } \\
\text { do de lado estilos decisorios o rasgos de personalidad, sin } \\
\text { embargo no se especifican cuales rasgos y cual es la ven- } \\
\text { taja de no retomarlos, esto es relevante considerando que } \\
\text { en el trabajo de Siebert y Kunz se menciona una "forma } \\
\text { de personar proactivamente" y se hace alusión al entre- } \\
\text { namiento de habilidades aún asociado con dichos rasgos. } \\
\text { Sería interesante que se ejemplificara cómo podría ser el } \\
\text { entrenamiento enfocándose en lo que sí está incluido den- } \\
\text { tro de la escala, o hacer mención de cómo funcionaría. }\end{array}$ \\
\hline \multicolumn{2}{|c|}{ Referencias } \\
\hline $\begin{array}{l}\text { Incrementar mas autores actuales y corregir las referen- } \\
\text { cias en el doi y recuperado de, quitar los dos putos }\end{array}$ & $\begin{array}{l}\text { Hay detalles en el formato como el uso de mayúsculas } \\
\text { en uno de los títulos y el espacio entre referencias que va } \\
\text { cambiando a lo largo del listado. }\end{array}$ \\
\hline
\end{tabular}


